\title{
Molecular analysis of EGFR gene in different types of tumor material from NSCLC patients
}

\author{
L. DOLESOVA ${ }^{1, *}$, M. KONECNY², J. MARKUS ${ }^{2}$, K. ZAVODNA $^{2}$, D. BOHMER ${ }^{1}$, V. REPISKA ${ }^{1}$ \\ ${ }^{1}$ Institute of Medical Biology, Genetics and Clinical Genetics, Faculty of Medicine and Faculty Hospital, Bratislava; ${ }^{2}$ Department of Clinical \\ Genetics, St. Elizabeth Cancer Institute, Bratislava \\ *Correspondence: dolesovalenka@gmail.com
}

Received August 5, 2014 / Accepted December 8, 2014

\begin{abstract}
Lung carcinoma is the most frequently occurring cancer worldwide and the Non-small Cell Lung Cancer (NSCLC) subtype represents $80 \%$ of all diagnosed cases. Epidermal growth factor receptor (EGFR) has an important dual role in NSCLC patients. On one hand, EGFR is frequently mutated in many types of tumors, which leads to deregulation of important downstream pathways including those affecting cell proliferation, differentiation and migration. On the other hand, presence of certain activating mutation leads to increased sensitivity of EGFR to tyrosine kinase inhibitors (TKIs) treatment. Detection of these mutations is essential for identification of NSCLC patients who would profit from such therapy. However, due to the nature of available tumor material and the relatively high number of mutation hot spots, such DNA analysis may be challenging and time consuming. Here we present an approach combining direct sequencing and SNaPshot assay for identification of EGFR mutations in FFPE tissues as well as in rarely analyzed cytological smears. Using this strategy on the set of 450 tested NSCLC samples; we have identified 29 activating mutations and 14 variants, which might be interesting in predicting the efficiency of TKI therapy.
\end{abstract}

Key words: non-small cell lung cancer, SNaPshot analysis, sequencing, EGFR mutations

Lung carcinoma is the most frequently occurring cancer worldwide and is responsible for one third of the deaths resulting from malignant diseases [1]. In Slovakia, the lung cancer is the second most common cancer (following the colon cancer). There are 21 new cases per 100000 inhabitants diagnosed each year (11 in men and 10 in women population) [2]. Primary lung cancer may be divided into two different histological groups: small cell lung cancer (SCLC) and non-small cell lung cancer (NSCLC). The latter group represents almost $80 \%$ of all cases. NSCLC can be further divided into several other subtypes: squamous (epidermoid), adeno, large cell, neuroendocrine carcinoma and special types of carcinomas with pleiomorphic, sarcomatoid or sarcomatous elements [3].

Lung cancer treatment depends on the tumor stage and type. Therapy of lung carcinomas is based on the combination of different approaches, especially surgery, chemotherapy and radiotherapy. In SCLC patients, chemotherapy using the anti-tumor cytotoxic drugs, e.g. platinum derivatives, taxol or cyclophosphamides is the most commonly used treatment. In advanced stages of NSCLC, a personalized biological treatment with TKIs or monoclonal antibodies may be used [4].
Application of the EGFR monoclonal antibodies (cetuximab, panitumumab) leads to efficient blocking of several signaling pathways, e.g. MAPK cascade [5]. TKI treatment (erlotinib, gefitinib, afatinib), is frequently used in patients with NSCLC and detected EGFR activating mutations [6]. TK inhibitors are small molecules that bind to the activation loop of cytoplasmic domain of EGFR receptor and suppress its activity by competing with binding of ATP. Gefitinib, a reversible competitive inhibitor [7], has an effect on autophosphorylation and downstream signaling of EGFR cascade and induces programmed cell death of malignant cells [8]. Erlotinib is a direct, selective, reversible inhibitor, which reduces the autophosphorylation of EGFR in tumor cells, inhibition of EGFR-dependent cell proliferation and blocks the cell cycle at the G1 phase [9]. The secondary objective of treatment with TKIs is increased survival (6-12 months), improved quality of life and relatively healthy individual profile [10]. Presence of activating somatic variants in the EGFR gene strongly correlates with the response to TKI treatment in NSCLC patients, mainly in adenocarcinomas. On the other hand, EGFR mutations were rarely detected in patients with SCLC and in other epithelial malignancies. 
The EGFR gene is localized on the $7 \mathrm{p} 11.2$ and comprises 28 exons. The protein consists of 3 functional domains: extracellular, transmembrane and cytoplasmic [11]. The cytoplasmic part (thyrosine kinase domain) is responsible for phosporylation of downstream targets and its autoregulation. The EGFR protein plays major role in many critical cellular processes during embryonic development; moreover it regulates metabolic and physiological pathways in different tissues and accordingly controls proliferation and differentiation of cells [12]. Mutations affecting EGFR activity have major impact on the regulation of cell proliferation and differentiation; and therefore are associated with many cancers. Most clinically important mutations are located in exons 18-21, which encode most of the TKI domain.

These mutations activate transforming potential of EGFR; however, they also make it more susceptible to inhibition by small-molecules - thyrosine kinase inhibitors (TKIs). Such increased sensitivity plays an important role and correlates with response to such therapy. The overall detection frequency of activating mutations varies from 7 to $15 \%$ in different studies. The exact frequency depends on the population origin, gender, smoking history and histological subtype of the tumor [13]. Mutations in EGFR are most common in patients of Asian origin (35-56\%), adenocarcinomas (13-47\%), women and nonsmokers [13]. Majority of activating mutations, approximately $90 \%$ occur in exons 19 and 21 [14].

Resistance to therapy is a major problem of lung cancer treatment. In most cases of the primary lung tumors it is caused by the overexpression of EGFR receptor. The secondary resistance is associated with the presence of p.T790M [15], p.L747S [16] and p.D761Y [17] substitutions, which predominantly occur in patients with positive primary response to treatment [18]. Balak et al. (2006) [17] reported point mutation p.T790M in more than $50 \%$ of patients treated with gefitinib, thus this substitution is considered to be the most common and has an inducible character [19].

As detailed below, detection of somatic mutations in clinical tumor samples may be a challenging task. Several studies reported detection of EGFR mutations in different types of material, such as plasma, native tissue and bronchoscopic cytological smears. However, the most frequently analyzed material was the formalin-fixed paraffin-embedded (FFPE) tumor tissue [20]. The biggest challenge of genetic DNA analysis from the tumor is low representation of tumor cells in the analyzed sample [21]. It is virtually impossible to collect only malignant cells without the surrounding normal tissue. The mutation is present only in tumor cells and not necessarily in all of them. Such "dilution" of mutated DNA may lead to false-negative results. Therefore, the quality of the analyzed material, especially in case of cytological smears is an important criterion and should be established for every sample. Cytological slides should contain at least 200-400 cells total and the proportion of tumor cells should be at least 50\% [21]. If the proportion of tumor cells is lower sensitivity of molecular analyses may be affected and false negative results may be obtained. Some molecular methods can be further inhibited by the presence of mucus or erythrocytes in sample.

Molecular methods for detection of mutations in tumors are often based on modified PCR approaches, such as COLDPCR, qPCR, HRM analysis or ARMS. These methods increase sensitivity by e.g. enriching the underrepresented mutated allele using the selective amplification [22]. The quantitative real-time PCR (qPCR) with specific fluorescence-labeled probes is one of the most frequently used methods nowadays. As the probes are sequence-specific, this method may reduce occurrence of false-positive results. However, use of separate probes for each mutant and WT allele is recommended, which increases the cost of reaction. Due to the limited number of usable fluorophores in the same well and the risk of crossreaction between probes also lower the ability to multiplex more variants in one reaction.

Melting analysis using the intercalating fluorescent dyes (e.g. SYBR Green) is much simpler and cheaper qPCR variant. High resolution melting assay (HRM) is based on the detection of differences between melting curves of PCR products from mutated and WT template [23]. Analysis has several advantages, such as minimal reagent and sample consumption and low cost [24]. The disadvantage of this approach is the "nonspecific" or indirect detection of mutations. This method will detect any variation present in the PCR product and positive results need to be confirmed by another sequence-specific methods, such as direct sequencing [21].

Other, less frequently used assays for detection of EGFR mutations and TKI sensitivity monitoring are EGFR array, DHPLC and MEL. EGFR array is based on the hybridization with sequence-specific probes [24]. DHPLC method uses liquid chromatography to detect variants in PCR products via heteroduplex separation and thereby requires additional demanding validation for every PCR fragment. Also, multiple test samples need to be analyzed for each clinical specimen [25]. MEL technology uses probes complementary to mutant and WT DNA and combines hybridization arrays with PCR technology and mutant allele enrichment. Enrichment of mutant allele is achieved by treating the sample with specific restriction enzyme, which depletes WT allele. This method has several advantages, including high sensitivity around 99\% and relatively short hands-on time [26].

$\mathrm{SNaPshot}$ analysis is a multiplex method that uses amplification by PCR, followed by addition of single fluorescence-labeled base to the primer and immediate termination. Due to this mechanism of analysis, it is also called a minisequencing analysis. The primers are designed just before the position of interest. Once the primer is annealed, polymerase incorporates single ddNTP and reaction is immediately terminated. Each ddNTP is labeled by different fluorescent dyes, which allows identification of specific incorporated base. Addition of artificial flanking extensions of different sizes to the 5 ' end of each primer allows differentiation between mutations and multiplexing of the analysis. The main advantages of this method are relatively high sensitivity (up to $98 \%$ ) and the 
possibility of multiplexing of 4 or more analyzed position in single run [27]. Thus relatively complex genotype information can be obtained at relatively low cost and in reasonable time. However, there are disadvantages associated with relatively long hands-on workflow and specificity for tested mutation sites only (i.e. there is no information about mutations in the region surrounding of the target position).

Finally, Sanger sequencing, which is frequently reported as a gold standard technique, represents another reliable method for identification of mutations. This approach provides complex information about all mutations in the particular PCR fragment of analyzed gene. However, direct sequencing detects mutation only if it is represented in at least $15-20 \%$ of the overall sample [28]. This remains insufficient for the molecular DNA analysis of tumor material, because of tumor heterogeneity and presence of normal tissue in specimen. Therefore, it is not ideal for prediction of biological treatment as a sole method.

In this report we present a DNA analysis of NSCLC tumor samples from bronchoscopical cytological smears, which are reported very rarely in the literature. We provide comparison with DNA analysis of tumor FFPE biopsies, followed by closer characteristics of cytological smears and their effective usage in clinical practice. Furthermore we present frequency of identified EGFR activating mutations in Slovak NSCLS patients, our detection approach combining $\mathrm{SNaPshot}$ analysis and direct sequencing focused on positives and negatives. Finally, we introduce a group of EGFR variants of unknown clinical significance, which may represent a promising target of TKIs therapy in some patients.

\section{Material and methods}

Tumor samples. Patient samples were collected and analyzed between $04 / 2010$ and $01 / 2014$. NSCLC tumors were investigated in the form of bronchoscopical smears on cytological slides $(n=327)$ or FFPE tissue blocks $(n=123)$. The group of 450 patients consisted of 95 women (21\%) and 355 men (79\%). Specimens with known histology were represented by squamous carcinomas, adenocarcinomas and large cell carcinomas. Majority of samples (cytological smears) were without histological specification (Not Otherwise Specified - NOS).

Quality of bronchoscopic smears and representation of tumor cells were assessed by specialized cytologist. In the case of FFPE samples, percentage of tumor cells and specification of histological subtype were determined by pathologist.

Mutation analysis. DNA was isolated using commercial kits, QIAamp ${ }^{\circ}$ NA Mini Kit (Qiagen) for bronchoscopical smears and BIOstic ${ }^{\circ}$ FFPE Tissue DNA Isolation Kit (MoBio Laboratories, Inc.) for FFPE block samples. PCR amplification of EGFR exons 18-21 was performed using 1x Maxima Hot Start Master Mix (Fermentas), $1 \mu \mathrm{M}$ primers (available upon request) and 90 ng of DNA.

Presence of mutations in exons 18-21 of EGFR gene was tested using the combination of SNaPshot and direct se- quencing analyses. Deletions in exon 19 (c.2237_2251del15, c.2237_2254del18), substitutions in exon 18 (p.G719S, p.G719C, p.G719A), 20 (p.S768I, p.T790M) and 21 (p.L858R, p.L861Q) were analyzed by SNaPshot assay. Insertions/deletions in exon 20 were tested by direct sequencing of entire exon, as we were unable to design high-quality SNaPshot primers in this location.

SNaPshot reactions were performed according to manufacturer's instructions using the SNaPshot Multiplex Ready Reaction Mix (Life Technologies) and mutation-specific primers (available upon request). Extension products were purified by alkaline phosphatase (Fermentas), products were separated by capillary electrophoresis (ABI PRISM 3130 Genetic Analyser; Applied Biosystems) and analyzed using the GeneMapper 3.0 software (Applied Biosystems).

Direct sequencing was performed according to manufacturer's instructions using Big Dye Terminator ${ }^{\bullet} \mathrm{Cycle}$ Sequencing Kit v1.1 (Applied Biosystems). Products were purified using the SigmaSpin ${ }^{\mathrm{TM}}$ Post Reaction Clean Up Columns (SIGMA Aldrich) and separated by capillary electrophoresis (ABI PRISM 3130 Genetic Analyzer; Applied Biosystems). The results were analyzed with Sequencing analysis software (Applied Biosystems) and InDelFinder software (Adinis).

\section{Results}

Detection rate of EGFR mutations. EGFR substitutions in hot-spot exons 18,20, 21 and deletions in exon 19 were analyzed using SNaPshot analysis and insertions in exon 20 were analyzed by direct sequencing. Activating EGFR mutations were detected in 29 NSCLC samples (6.4\%), with $13.6 \%$ detection rate in female samples and $4.5 \%$ in males. The most frequently detected mutations were substitution p.L858R (48.3\%) in exon 21 and deletions c.2237_2251del15, c.2237_2254del18 in exon 19 (38\%). Other activating mutations were identified with lower frequencies: p.G719S in exon 18 in 2 cases (6.9\%), and p.S768I, p.T790M (exon 20), p.L861Q (exon 21) each in 1 case (3.4\%) (Table 1). We have also identified a rare, so far unreported mutational event in

Table 1. Overall count of identified EGFR activating mutation.

\begin{tabular}{lccc}
\hline Mutation type & Exon & Samples & \% \\
\hline p.G719S & 18 & 2 & 6,9 \\
\hline c.2237_2251del15 & \multirow{2}{*}{19} & 6 & 20,7 \\
c.2237_2254del18 & & 5 & 17,3 \\
\hline p.T790M & \multirow{2}{*}{20} & 1 & 3,4 \\
p.S768I & \multirow{2}{*}{21} & 1 & - \\
\hline p.L861Q & 1 & 3,4 \\
p.L858R & & 14 & 48,3 \\
\hline Summary & $29^{*}$ & 100 \\
\hline
\end{tabular}

* Comment: In one sample two somatic mutations were detected (p.S768I, p.L858R). 
EGFR gene, a male sample carrying two different activating EGFR mutations, p.S768I and p.L858R.

DNA analysis of different tumor material. We have tested 450 samples comprised of 327 bronchoscopical slides $(72.7 \%)$ and 123 FFPE tumor biopsies (27.3\%) for the presence of somatic mutations in exons 18-21 of the EGFR gene. Based on the histological typing, the samples were divided into 6 groups. The most abundant was the group of carcinomas without specification (NOS, $\mathrm{n}=198,44 \%$ ), followed by squamous cell carcinomas $(n=127,28.2 \%)$ and adenocarcinomas $(n=104$, 23.1\%). Remaining minor NSCLC types were represented by large-cell carcinomas $(n=5)$ and mixed squamous-adenocarcinomas $(n=3)$ (Table 2$)$.

In the set of FFPE tumor biopsies we identified 18 EGFR mutations (14.6\%, out of 123). Before analyzing mutations in the set of bronchoscopical cytological smear samples, we divided it into 3 groups based on the representation of malignant cells: with less than $50 \%$ of tumor cells on the slide $(n=101)$, over $50 \%$ of tumor cells $(n=180)$, and samples with undetermined tumor cell count (ND, $\mathrm{n}=46)$. As expected, all 11 EGFR mutations identified in cytological samples $(3.4 \%$, out of 327 ) were found in the group with over $50 \%$ tumor cells representation (Table 3 ).

Table 2. Clinical and histological characteristics of analyzed NSCLC patients group.

\begin{tabular}{llcc}
\hline Patient's gender & Male $(M)$ & \multicolumn{2}{c}{$355(78.9 \%)$} \\
$N$ (percent) & Female $(F)$ & $95(21.1 \%)$ \\
\hline EGFR status & WT & $421(93.6 \%)$ \\
$N$ (percent) & Mutated & $29(6.4 \%)$ \\
\hline Mutated samples gender & $M$ & \multicolumn{2}{c}{$16(4.5 \%)$} \\
$N$ (percent) & $F$ & \multicolumn{2}{c}{$13(13.7 \%)$} \\
\hline Histological subtypes & Adenocarcinoma & $M$ & $68(15.1 \%)$ \\
$N$ (percent) & & $F$ & $36(8 \%)$ \\
\cline { 2 - 4 } & Squamous carcinoma & $M$ & $121(26.9 \%)$ \\
& & $F$ & $19(4.2 \%)$ \\
\cline { 2 - 4 } & Large cell carcinoma & $M$ & $4(0.9 \%)$ \\
& & $F$ & $1(0.2 \%)$ \\
\cline { 2 - 4 } & Squamous adenocarcinoma & $M$ & $3(0.7 \%)$ \\
& & $F$ & - \\
\cline { 2 - 4 } & NOS & M & $165(36.7 \%)$ \\
& & $F$ & $33(7.3 \%)$ \\
\hline
\end{tabular}

Table 3. Overview of types of analyzed material and representation of tumor cells.

\begin{tabular}{llccc}
\hline & & $\begin{array}{c}\text { Content of } \\
\text { tumor cells }\end{array}$ & N(percent) & $\begin{array}{c}\text { Mutated } \\
\text { samples }\end{array}$ \\
\hline \multirow{3}{*}{$\begin{array}{l}\text { Type of } \\
\text { material }\end{array}$} & Cytological & to $50 \%$ & $101(21.2 \%)$ & \\
\cline { 2 - 5 } & samples & over $50 \%$ & $180(37.7 \%)$ & $11(3.4 \%)$ \\
\cline { 2 - 5 } & indetermined & $46(9.6 \%)$ & \\
\hline
\end{tabular}

SNaPshot analysis of activating EGFR mutations. For the detection of deletions in exon 19, we used 2 pairs of SNaPshot primers designed exactly before the breakpoints of the deletion c.2237_2251del15 and c.2237_2254del18 (Figure 1). Using this concept we identified 6 samples with 15-bp and 5 samples with 18-bp deletion. Mutations in exon 18, 20 and 21 were detected using forward and reverse SNaPshot primers to increase specificity and sensitivity of the approach (Figure 1). Thus we identified p.L858R mutation in 14 samples, p.S768I and the resistance-associated p.T790M in 2 samples each and p.G719S in one case. The presence of these mutations was confirmed by Sanger sequencing in selected samples (Figure 2).

Sequencing analysis of EGFR activating mutations. EGFR exon 20 is known to be susceptible for the occurrence of activating insertions. As opposed to exon 19 and due to the insertions localization, we were not successful to design SNaPshot primers for these mutations. Consequently, the presence of insertions in exon 20 was analyzed only by direct sequencing approach, however no insertion or deletion mutation was identified.

On the other hand, during the validation of our SNaPshot design, we performed Sanger sequencing on all 4 exons of interest in selected samples $(n=145)$. Using this approach, we identified several variants of uncertain clinical significance (VUS) in exons 19, 20 and 21 of EGFR gene. Altogether 14 different variant samples (9.7\%) were present in 145 sequenced specimens. Due to insufficient published information on these variants, we performed an in silico analysis of possible impact of these variants on the EGFR protein. We used two different tools available online - Polyphen and AGVGD (Table 4). Our results suggest that at least some of these mutations might be of clinical significance and should be further investigated.

Table 4. The effect of identified EGFR variants predicted using the online tools Polyphen and AGVGD and their possible clinical significance. In AGVGD prediction class C65 is supposed to be most likely pathogenic and $\mathrm{CO}$ less likely pathogenic.

\begin{tabular}{lccc}
\hline Variant & EGFR exon & Polyphen prediction (probability) & AGVGD \\
\hline p.I732T & Poss. Damaging $(0,855)$ & $C 65$ \\
p.K739E & Prob. Damaging $(1,000)$ & $C 65$ \\
p.P741L & Prob. Damaging $(1,000)$ & $C 65$ \\
p.V742A & \multirow{2}{*}{ Prob. Damaging $(1,000)$} & $C 65$ \\
p.A743L & & Prob. Damaging $(1,000)$ & $C 65$ \\
p.I744T & Prob. Damaging $(1,000)$ & $C 65$ \\
p.R748G & & Poss. Damaging $(0,981)$ & $C 65$ \\
p.P753S & & Poss. Damaging $(0,949)$ & $C 65$ \\
\hline p.C775Y & & Prob. Damaging $(1,000)$ & $C 65$ \\
p.I780T & & Prob. Damaging $(1,000)$ & $C 65$ \\
p.L782P & 20 & Poss. Damaging $(0,888)$ & $C 65$ \\
p.S784P & & Benign (0,045) & $C 65$ \\
p.L788I & & Prob. Damaging $(1,000)$ & $C 0$ \\
\hline p.D800N & 21 & Prob. Damaging $(0,996)$ & $C 15$ \\
\hline
\end{tabular}




\section{Discussion}

Frequency of activating EGFR mutations (6.4\%) detected in our patients group is in concordance with general reported detection rates, which are between 5-15\% [29]. Another study of EGFR mutations in non-Asian population detected their frequency at about $6 \%$ [30]. On the other hand, some studies demonstrated much higher estimated EGFR mutation frequencies, at $10 \%, 18.6 \%$ and $28.1 \%$ respectively $[31,26,32]$. There are very few papers reporting the DNA analysis from
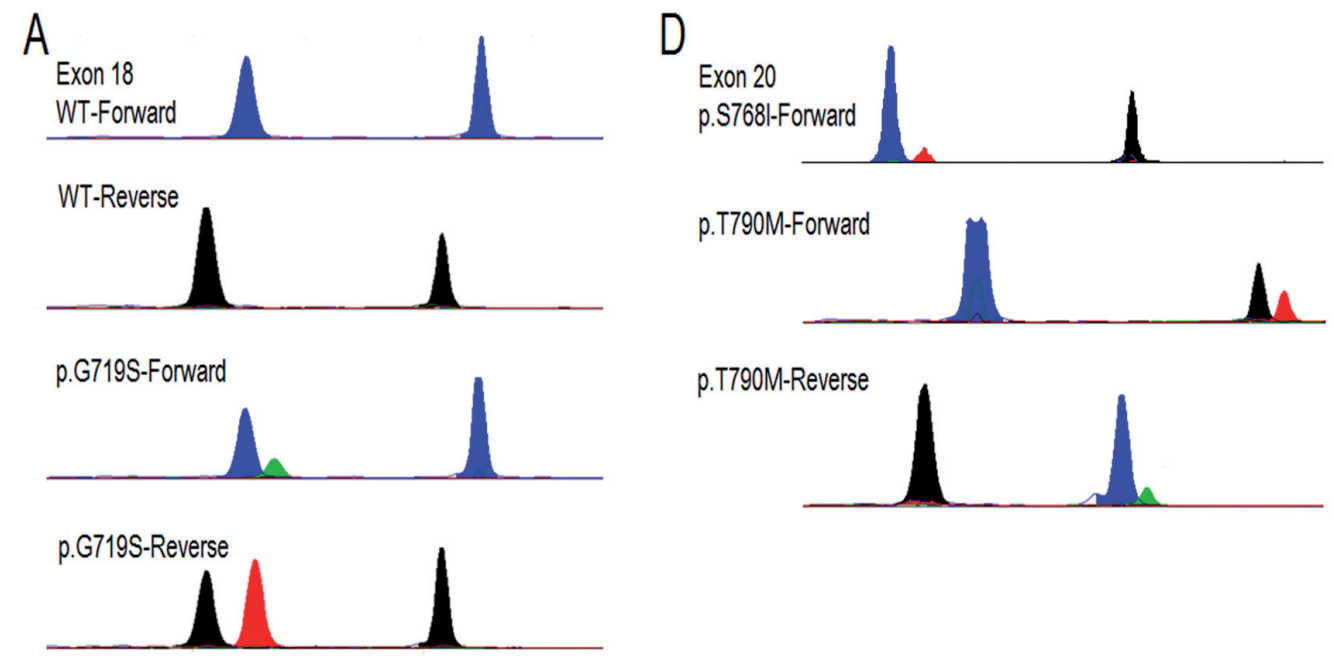

\section{p.T790M-Reverse}

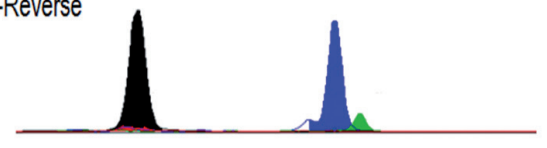

B

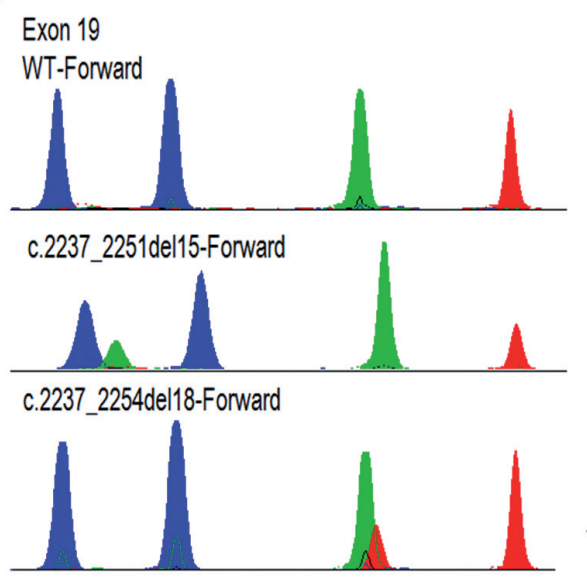

C

E

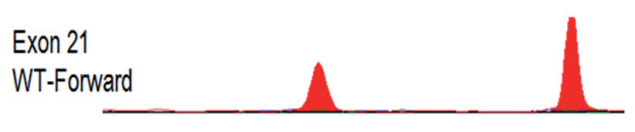

WT-Reverse

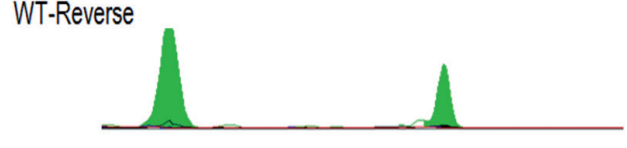

p.L858R-Forward

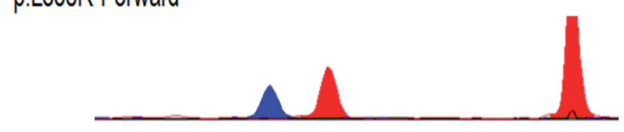

p.L858R-Reverse

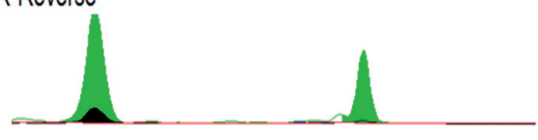

Exon 20

WT-Forward

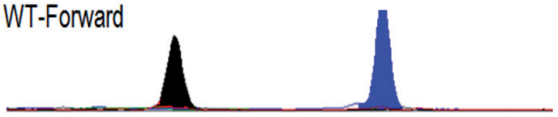

WT-Forward

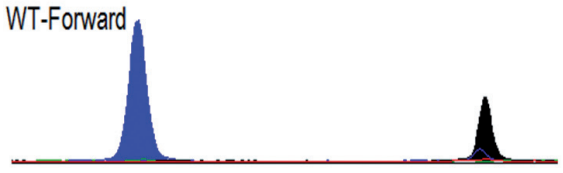

p.L861Q-Forward

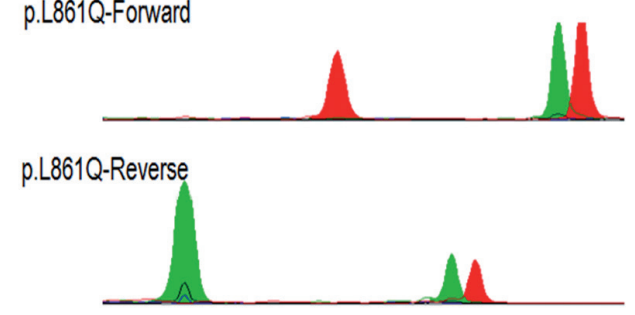

Figure 1. Electrophoretograms of SNaPshot analysis. A: Exon 18, wild-type sample in comparison with p.G719S sample, B: Exon 19, wild-type sample and deletions in exon 19, C, D: Exon 20, wild-type sample in comparison with mutations p.S768I and p.T790M, E: Exon 21, wild-type and the most common mutation p.L858R and p.L861Q. 
A

Exón 19

WT-Forward

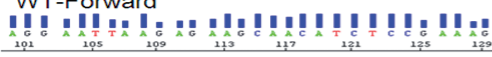

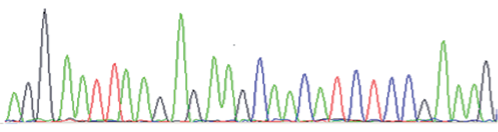

c.2237_2251del15-Forward

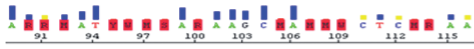

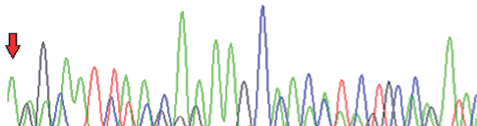

c.2237_2254del18-Forward

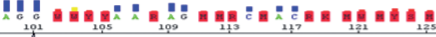

,

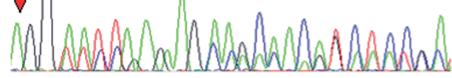

B

Exón 20

WT-Forward

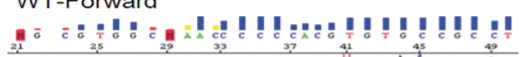

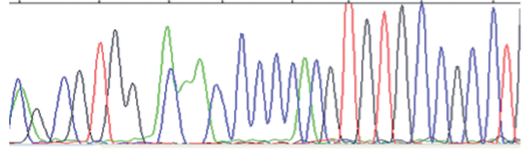

p.S768I-Forward

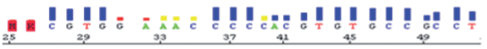

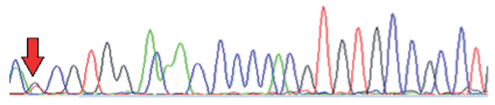
WT-Forward

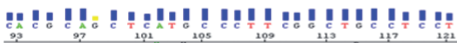

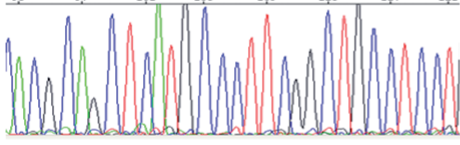

p.T790M-Forward

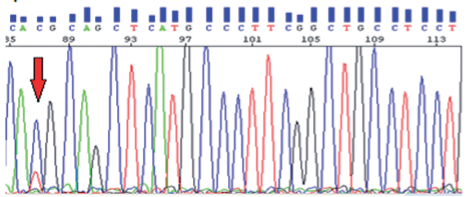

C

Exón 21

WT-Forward

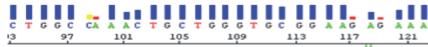

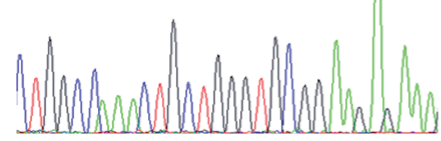

p.L858R-Forward

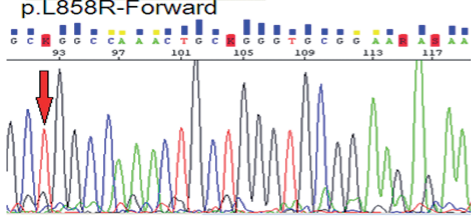

WT-Reverse

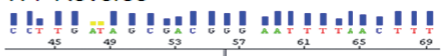

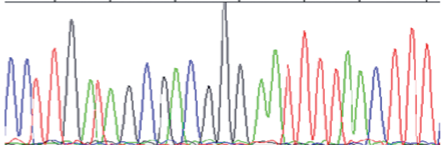

c.2237_2251del15-Reverse

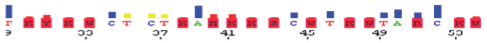

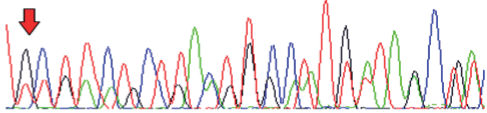

c. 2237 2254del18-Reverse

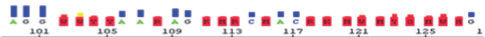

ת

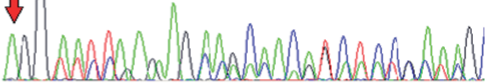

WT-Reverse

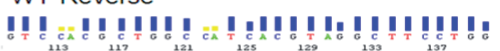

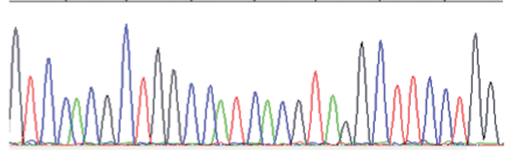

p.S768I-Reverse

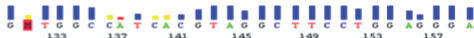

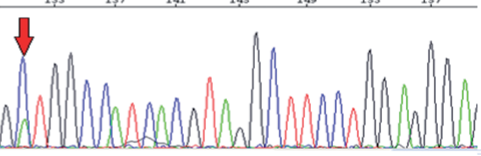

WT-Reverse

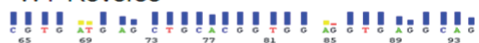

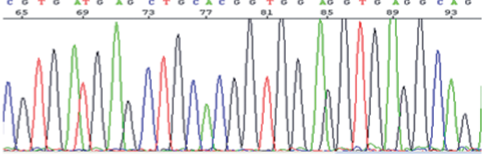

p. T790M-Reverse

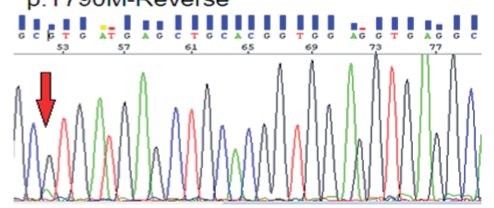

WT-Reverse
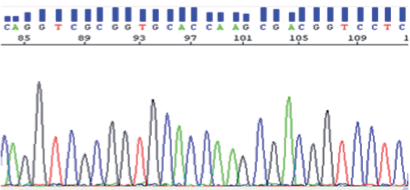

p.L858R-Reverse

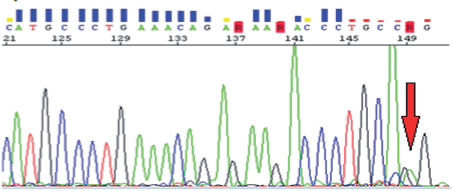

Figure 2. Sequencing analysis electrophoretograms of selected samples. A: Exon 19, wild type and specimens with 15 bp, 18 bp deletion, B: Substitutions p.S768I and p.T790M in exon 20, C: Substitution p.L858R in exon 21. 
cytological smears or discussing such specimens, which are often the only available material for lung tumors. Conversely, FFPE tumor samples are generally considered as standard material for EGFR mutation detection. Therefore we assume that the final mutation detection ratio in our study is mainly influenced by the quantity and quality of input tumor material. On the other hand, factors such as structure of analyzed sample group in the terms of gender and tumor histology, the status of patient's treatment (before or after TKI treatment) should be taken into the account.

Based on the literature data, deletions in exon 19 and substitution p.L858R in exon 21 are the most frequently occurring mutations in NSCLC samples (80-90\%), independently on the histological subtype [33]. This finding was confirmed in our samples as well. These two mutations represented $86.3 \%$, specifically, out of 29 patients with mutations, 12 patients carried p.L858R (48.3\%) and 11 patients carried deletions in exon 19 (38\%). Mutations in other exons represented only $13.7 \%$, specifically we identified 2 cases of p.G719S in exon 18 and 1 case each of p.T790M, p.S768I in exon 20 and p.L861Q in exon 21.

EGFR mutations are most frequently detected in women, non-smokers with adenocarcinoma and in patients from Asian population [34]. Altogether, in the group of 450 NSCLC patients, we detected the presence of EGFR mutations in 3 types of NSCLC with highest detection rate in adenocarcinomas (9.6\%), followed by NOS (8.1\%) and squamous cell carcinoma (2.3\%), which correlates with previously reported data [25]. Interestingly, when we divided adenocarcinomas according to the gender, the frequency of activating mutation was almost two times higher (11\%) in female adenocarcinomas.

As reported in literature, generally preferred tumor material for molecular EGFR analysis and detection of somatic mutations is FFPE tumor tissue $[35,36]$. However, in case of lung tumors, availability of such material may be limited due to tumor location and many technical limitations. For these patients only cytological smears may be available. In our study, majority of the samples were represented by cytological bronchoscopical smears (72.7\%), which are not ideal input material for DNA analysis. This is supported by the fact, that we have found only few previous studies that use the same material [31]. In our study we found EGFR mutations only in 3.4\% of these specimens ( 11 of 327 ), that may indicate poor quality and quantity of analyzed DNA. Also, many of these samples have undetermined percentage of malignant cells mainly due to the small size of specimen. The usage of cytological bronchoscopical smears in clinical practice is thus controversial and should be based on strict quality criteria.

The detection rate in the group of FFPE samples was much higher, represented by $14.6 \%$ of mutated samples (18 of 123), suggesting that use of this material for DNA analysis is much more effective. Angulo et al. [35] and Marchetti et al. [36] analyzed FFPE material and reported mutation frequencies of $16.2 \%$ and $24.1 \%$ respectively, a detection rate relatively close to our FFPE set. Therefore we assume that our mutation detection approach is not the reason why such a low mutation frequency was detected in cytological smear samples. The main factors affecting DNA analysis may be lower representation of tumor cells, lower quality of isolated tumor DNA, lower total number of cells, method and aggressiveness of fixation, artifacts of cytological staining and even bleaching before DNA isolation. This conclusion was supported by the analysis of 26 parallel tumors for which we had both cytological smears and FFPE tissues available. In this set of samples we have identified p.L858R mutation in 2 FFPE samples; however, no mutation was detected in corresponding cytological slides. According to all these facts, we suggest that cytological bronchoscopical smears should be considered suitable for DNA analysis only when strict qualitative criteria are fulfilled, mainly total cell count and percentage of tumor cells. Also size of the tumor sample is an important parameter affecting genetic analysis from bronchoscopic biopsies and smears. These samples are often small and malignant cells may represent only a little fraction and therefore affect proper histological definition of the tumor as well [20].

Histological determination of cytological specimens may be also very difficult, which is confirmed by high number of cytological samples with no histological specification (NOS) in our study (approximately $44 \%$ of our specimens). Surprisingly, we determined relatively high mutation frequency $(8.1 \%)$ in this group, suggesting that a many of NOS samples may be in reality "hidden" adenocarcinomas. The most commonly occurring known histological type was squamous cell carcinoma (28.2\%) followed by adenocarcinoma (23.1\%). In most reported studies, NOS carcinomas usually represent less than $10 \%$ (3.9\%, respectively $8.1 \%)$ of analyzed samples [37, $38]$ and adenocarcinomas are usually the largest analyzed group of samples ( $70 \%, 81.1 \%$ or $97.7 \%$, respectively) $[37,38$, 39]. However, this is also associated with the fact that other studies primarily utilize FFPE material. Histologization is not so precise in cytological samples, which represented majority in our group.

Recent study by Hlinkova et al. reported results from twoyear experience of EGFR somatic mutation detection from cytological samples. Similarly to our results, authors detected lower frequency of activating mutations at the level of $10 \%$; however they declare very good cellularity and percentage of tumor cells in specimens [31].

In our study we detected a very rare mutational event in EGFR gene, which to our knowledge, was not reported in the literature so far. We have identified presence of two activating mutations p.L858R and p.S768I in one sample, which were confirmed by both SNaPshot analysis and Sanger sequencing. The DNA sample was isolated from FFPE tissue of male patient and paradoxically with declared squamous (epidermoid) cell carcinoma. Unfortunately, we were unable to obtain clinical data about TKI therapy of this patient. We may speculate that this patient had greater-than-usual benefit from biological treatment. Although some studies have reported cases of double mutants, they always involved combination 
one activating mutation and one resistance mutation $[35,40]$. Double mutants are usually only found in cell lines, especially substitutions in exon 21 (p.L858R) and in exon 20 (p.T790M). However, presence of resistance mutation p.T790M is generally initiated after TKI treatment [16].

Angulo et al. who analyzed 133 samples, reported that sequencing analysis has lower sensitivity than SNaPshot. In the case of direct sequencing, mutated sequence may be confused with background noise due to the low signal intensity. Thereby may lead to false negative results mainly in the samples with lower representation of mutated DNA. Direct sequencing was able to detect the presence of an EGFR mutation when mutant DNA represented at least $10 \%$ of the total DNA [35]. It means that the use of direct sequencing, as an individual method, requires better quality of samples and higher proportion of tumor cells. We used sequencing analysis only for detection of insertions in EGFR exon 20, but in our set we did not find any such mutations. On the other hand, the insertions in exon 20 occur at very low frequency of $2.5 \%$ [41]. Thus this finding is not unexpected and sequencing still may be considered as appropriate, but only as supplementary method. Our attempt to solve the problem with low sensitivity of direct sequencing by SNaPshot was not successful. Mutation hot-spots in exon 20 are localized close to each other and are surrounded by "problematic" sequences, so the primers with appropriate parameters could not be designed.

$\mathrm{SNaPshot}$ assay as a mutation specific detection method is quite frequently used approach for mutation hot-spots in various genes. In comparison with Sanger sequencing, it represents relatively simple and inexpensive method, however detects only known variations in particular gene. Clearly, one limitation of SNaPshot assay is that it does not reveal information about all mutations in the entire exon. However, it can be used to detect mutations in cases with low proportion of mutant DNA as the declared sensitivity is at level 1.56-12.5\%. Most importantly, this method is reported as robust, reliable, rapid and relatively inexpensive [27].

During the validation stage of $\mathrm{SNaPshot}$ analysis we used Sanger sequencing of all 4 exons in selected 145 samples as a confirmation method. During this process we detected several unreported and rare variants of EGFR gene in 14 samples (3.1\%). Due to the lack of published information about $E G F R$ variants of uncertain significance we performed an in silico prediction using the freely available online tools Polyphen and AGVGD. To test the prediction value in the case of known activating mutation we analyzed also p.L858R. According to the analysis results (Table 4), eight of these 8 VUS were labeled as probably damaging by both tools and 2 as possibly damaging. Similar values were calculated in the case of p.L858R mutation, whose carriers are known to benefit from biological treatment with EGFR TKIs. These results may indicate that use of sequencing analysis may reveal presence of rare VUS, which would uncover patients potentially benefiting from TKI therapy. Naturally, further analysis of these mutations and the therapy outcomes of such mutations carriers are necessary. This would however depend on the interest and compliance from clinicians and healthcare insurances.

In conclusion, our study demonstrates the detection of EGFR activating mutations using a combination of $\mathrm{SNaPshot}$ analysis and direct sequencing in the two different types of material - cytological bronchoscopical smears and FFPE tissue samples. Our results show, that usability of cytological smears is limited and reliable only if the sample contains at least $50 \%$ of tumor cells. Absolute number of tumor cells should be considered as well. Combination of presented methods represents an effective and relatively rapid 'homemade' approach, with outcome comparable to available commercial techniques. This is further confirmed by the fact that the spectrum and types of detected mutations are also very similar to other published studies. Interestingly, we identified a sample with very rare occurrence of two different activating mutations in one patient as well as 14 different rare or novel uncertain variants in EGFR gene.

\section{References}

[1] JEMAL A, SIEGEL R, WARD E, HAO Y, XU J et al. Cancer statistics, 2008. CA Cancer J Clin 2008; 58: 71-96. http:// dx.doi.org/10.3322/CA.2007.0010

[2] FERLAY J, SHIN H, BRAY F, FORMAN D, MATHERS C et al. Estimates of worldwide burden of cancer in 2008: GLOBOCAN 2008. IJC 2010; 127 : 2893-2917.

[3] PAO W, GIRARD N. New driver mutations in non-small-cell lung cancer. Lancet Oncol 2011; $12:$ 175-80. http://dx.doi. org/10.1016/S1470-2045(10)70087-5

[4] PINES G, KOSTLER W J, YOSEF YARDEN department of biological regulation, the weizmann institute of science, rehovot 76100, ISRAEL. Oncogenic mutant forms of EGFR: lessons in signal transduction and targets for cancer therapy. FEBS Lett. 2010; 584 :2699-2706. http://dx.doi.org/10.1016/j. febslet.2010.04.019

[5] MITSUDOMI T, YATABE Y. Mutations of the epidermal growth factor receptor gene and related genes as determinants of epidermal growth factor receptor tyrosine kinase inhibitors sensitivity in lung cancer. Cancer Science 2007; 98 : 1817-1824. http://dx.doi.org/10.1111/j.1349-7006 2007.00607.x

[6] ROSELL R, MORAN T, QUERALT C, PORTA R, CARDENAL F et al. Screening for epidermal growth factor receptor mutations in lung cancer. N Engl J Med. 2009; 361: 958-967. http://dx.doi.org/10.1056/NEJMoa0904554

[7] BASELGA J, AVERBUCH SD. ZD1839 (,Iressa') as an anticancer agent Drugs. 2000; 60 : 33-40 http://dx.doi. org/10.2165/00003495-200060001-00004

[8] JIANG J, GREULICH H, JÄNNE PA, SELLERS WR, MEYERSON $\mathrm{M}$ et al. Epidermal growth factor independent transformation of $\mathrm{Ba} / \mathrm{F} 3$ cells with cancer-derived epidermal growth factor receptor mutants induces gefitinib-sensitive cell cycle progression. Cancer Res. 2005; 65 : 8968-74 http:// dx.doi.org/10.1158/0008-5472.CAN-05-1829 
[9] POLLACK VA, SAVAGE DM, BAKER DA, TSAPARIKOS $\mathrm{KE}$, SLOAN DE et al. Inhibition of epidermal growth factor receptorassociated tyrosine phosphorylation in human carcinomas with CP-358,774: Dynamics of receptor inhibition in situ and antitumor effects in athymic mice. J Pharmacol Exp Ther. 1999; 291: 739-748

[10] PEREZ-SOLER R, CHACHOUA A, HAMMOND LA, ROWINSKY EK, HUBERMAN $M$ et al. Determinants of Tumor Response and Survival With Erlotinib in Patients With Non-Small-Cell Lung Cancer. J Clin Oncol 2004; 22: 16 http:// dx.doi.org/10.1200/JCO.2004.11.057

[11] ROBERTS SK, TYNAN CHJ, WINN M, MARTIN-FERNANDEZ ML. Investigating extracellular in situ EGFR structure and conformational changes using FRET microscopy. Biochem Soc Trans 2012; 40: 189-194. http://dx.doi.org/10.1042/ BST20110632

[12] BLUME-JENSEN P, HUNTER T. Nature 2001; 411: 355-365. http://dx.doi.org/10.1038/35077225

[13] SEKINE I, YAMAMOTO N, NISHIO K, SAIJO N. Emerging ethnic differences in lung cancer therapy. Br J Cancer 2008: 1757-1762. http://dx.doi.org/10.1038/sj.bjc.6604721

[14] NGUYEN KS, KOBAYASHI S, COSTA DB. Acquired resistance to epidermal growth factor receptor tyrosine kinase inhibitors in non-small-cell lung cancers dependent on the epidermal growth factor receptor pathway. Clinical lung cancer 2009; 10: 281-289. http://dx.doi.org/10.3816/CLC.2009.n.039

[15] KOBAYASHI S, BOGGON TJ, DAYARAM T, JANNE PA, KOCHER $\mathrm{O}$ et al. EGFR mutation and resistance of nonsmall-cell lung cancer to gefitinib. N Engl J Med. 2005; 352 : 786-792 http://dx.doi.org/10.1056/NEJMoa044238

[16] COSTA DB, HALMOS B, KUMAR A, SCHUMER ST, HUBERMAN MS et al. Bim mediates egfr tyrosine kinase inhibitor-induced apoptosis in lung cancers with oncogenic egfr mutations. PLoS Med. 2007; 4: 1669-1679 http://dx.doi. org/10.1371/journal.pmed.0040315

[17] BALAK MN, GONG Y, RIELY GJ, SOMWAR R, LI AR et al. Novel d761y and common secondary t790m mutations in epidermal growth factor receptormutant lung adenocarcinomas with acquired resistance to kinase inhibitors. Clin Cancer Res. 2006; 12: 6494-6501 http://dx.doi.org/10.1158/1078-0432. CCR-06-1570

[18] BURRIS HA. Shortcomings of current therapies for nonsmallcell lung cancer: Unmet medical needs. Oncogene 2009; 28: S4-13 http://dx.doi.org/10.1038/onc.2009.196

[19] PAO W, MILLER VA, POLITI KA, RIELY GJ, SOMWAR R et al. Acquired resistance of lung adenocarcinomas to gefitinib or erlotinib is associated with a second mutation in the EGFR kinase domain. PLoS Med. 2005; 2: e73 http://dx.doi. org/10.1371/journal.pmed.0020073

[20] TRAVIS WD, BRAMBILLA E, NOGUCHI M, NICHOLSON A, GEISINGER K et al. Diagnosis of Lung Cancer in Small Biopsies and Cytology Implications of the 2011 International Association for the Study of Lung Cancer/ American Thoracic Society/European Respiratory Society Classification. Arch Pathol Lab Med. 2011; 136: 1-17.

[21] LI J, MAKRIGIORGOS G M. COLD-PCR: a new platform for highly improved mutation detection in cancer and genetic testing. Biochem Soc Trans 2009; 37: 427-32. http://dx.doi. org/10.1042/BST0370427

[22] REED, GH, KENT JO, WITTWER CT. High-resolution DNA melting analysis for simple and efficient molecular diagnostics. Pharmacogenomics 2007; 8: 597-608. http://dx.doi. org/10.2217/14622416.8.6.597

[23] HOLDEN JA, WILLMORE-PAYNE C, COPPOLA D, GARRETT CR, LAYFIELD LJ. High-resolution melting amplicon analysis as a method to detect c-kit and platelet-derived growth factor receptor alpha activating mutations in gastrointestinal stromal tumors. Am J Clin Pathol. 2007; 128: 230-238. http://dx.doi.org/10.1309/7TEH56K6WWXENNQY

[24] YAM I, LAM DC, CHAN K, CHUNG-MAN HJ, IP M et al. EGFR array: uses in the detection of plasma EGFR mutations in non-small cell lung cancer patients. J Thorac Oncol 2012; 7(7): 1131-40. http://dx.doi.org/10.1097/ JTO.0b013e3182558198

[25] WOLFORD J, BLUNT D, BALLECER C, PROCHAZKA M. High-throughput SNP detection by using DNA pooling and denaturing high performance liquid chromatography (DHPLC). Hum Genet 2000; 107: 483-487. http://dx.doi. org/10.1007/s004390000396

[26] AN SJ, CHEN ZH, SU J, ZHANG XCH, ZHONG WZ et al. Identification of Enriched Driver Gene Alterations in Subgroups of Non-Small Cell Lung Cancer Patients Based on Histology and Smoking Status. PLOS ONE 2011; 7 : 40109.

[27] SU Z, DIAS-SANTAGATA D, DUKE M, HUTCHINSON K, LIN YL et al. A Platform for Rapid Detection of Multiple Oncogenic Mutations With Relevance to Targeted Therapy in Non-Small-Cell Lung Cancer. J Mol Diagn. Jan 2011; 13: 74-84. http://dx.doi.org/10.1016/j.jmoldx.2010.11.010

[28] METZKER ML. Emerging technologies in DNA sequencing. Genome Res 2005; 15: 1767-1776. http://dx.doi.org/10.1101/ gr.3770505

[29] LYNCH TJ, BELL DW, SORDELLA R, GURUBHAGAVATULA S, OKIMOTO RA et al. Activating mutations in the epidermal growth factor receptor underlying responsiveness of non-small cell lung cancer to gefitinib. N Engl J Med 2004; 350: 2129-2139. http://dx.doi.org/10.1056/NEJMoa040938

[30] SHIGEMATSU H, GAZDAR AF. Somatic mutations of epidermal growth factor receptor signaling pathway in lung cancers. Int J Cancer 2006; 118: 257-262. http://dx.doi. org/10.1002/ijc. 21496

[31] HLINKOVA K, BABAL P, BERZINEC P, MAJER I, MIKLEBARATHOVA $Z$ et al. Evaluation of two-year experience with EGFR mutation analysis of small diagnostic samples. Diagn Mol Pathol. 2013; 22: 70-5. http://dx.doi.org/10.1097/ PDM.0b013e31827e6984

[32] MURRAY S, KARAVASILIS V, BOBOS M, RAZIS E, PAPADOPOULOS S et al. Molecular predictors of response to tyrosine kinase inhibitors in patients with Non-Small-Cell Lung Cancer. J Exp Clin Cancer Res 2012; 31: 77. http://dx.doi. org/10.1186/1756-9966-31-77

[33] MITSUDOMI T, YATABE Y. Mutations of the epidermal growth factor receptor gene and related genes as determinants of epidermal growth factor receptor tyrosine kinase inhibitors 
sensitivity in lung cancer. Cancer Science 2007; 98: 1817-1824 http://dx.doi.org/10.1111/j.1349-7006.2007.00607.x

[34] PAEZ JG, JANNE PA, LEE JC, TRACY S, GREULICH H et al. EGFR mutations in lung cancer: correlation with clinical response to gefitinib therapy. Science 2004; 81: 61-9.

[35] ANGULO B, CONDE E, SUAREZ-GAUTHIER A, PLAZA C, MARTINEZ R et al. A Comparison of EGFR Mutation Testing Methods in Lung Carcinoma: Direct Sequencing, Real-time PCR and Immunohistochemistry. PLOS ONE 2012; 7: 43842. http://dx.doi.org/10.1371/journal.pone.0043842

[36] MARCHETTI A, MILELLA M, FELICIONI L, CAPPUZZO F, IRTELLI L et al. Clinical Implications of KRAS Mutations in Lung Cancer Patients Treated with Tyrosine Kinase Inhibitors: An Important Role for Mutations in Minor Clones. Neoplasia 2009; 11: 1084-1092.

[37] SONOBE M, MANABE T, WADA H, TANAKA F. Mutations in the epidermal growth factor receptor gene are linked to smoking-independent, lung adenocarcinoma. Br J Cancer 2005; 93: 355-363. http://dx.doi.org/10.1038/sj.bjc.6602707
[38] KELLY RJ, RAJAN A, FORCE J, LOPEZ-CHAVEZ A, KEEN $\mathrm{C}$ et al. Evaluation of KRAS mutations, angiogenic biomarkers and DCE-MRI in patients with advanced non-small cell lung cancer receiving sorafenib. Clin Cancer Res 2011; 17 (5): 1190-1199. http://dx.doi.org/10.1158/1078-0432.CCR10-2331

[39] TANIGUCHI K, UCHIDA J, NISHINO K, KUMAGAI T, OKUYAMA T et al. Quantitative Detection of EGFR Mutations in Circulating Tumor DNA Derived from Lung Adenocarcinomas. Clin Cancer Res 2011; 17 (24): 7808-15. http://dx.doi.org/10.1158/1078-0432.CCR-11-1712

[40] BORRAS E, JURADO I, HERNAN I, GAMUNDI M J, DIAS $\mathrm{M}$ et al. Clinical pharmacogenomic testing of KRAS, BRAF and EGFR mutations by high resolution melting analysis and ultra-deep pyrosequencing. BMC Cancer 2011; 11: 406. http:// dx.doi.org/10.1186/1471-2407-11-406

[41] SHARMA SV, SETTLEMAN J. Erbbs in lung cancer. Exp Cell Res 2009; 315: 557-571. http://dx.doi.org/10.1016/j. yexcr.2008.07.026 